\title{
Train model acceleration and deceleration
}

\author{
YANG QianSuo*, SONG JunHao, LI Duo, ZHANG Jie \& YANG GuoWei \\ State Key Laboratory of High Temperature Gas Dynamics, Institute of Mechanics, Chinese Academy of Sciences, Beijing 100190, China
}

Received October 7, 2012; accepted December 3, 2012; published online January 10, 2013

\begin{abstract}
In order to accelerate a heavy train model with great dimensions to a speed higher than $300 \mathrm{~km} \mathrm{~h}^{-1}$ in a moving train model testing system, compressed air is utilized to drive the train model indirectly. The gas from an air gun pushes the piston in an accelerating tube forward. The piston is connected to the trailer through a rope, and the trailer pulls the train model to the desired speed. After the testing section, the train model enters the deceleration section. The speed of the train model gradually decreases because of the braking force of the magnetic braking device on the bottom of the train model and the steel plates fixed on the floor of this device. The dissipation of kinetic energy of the trailer is also based on a similar principle. The feasibility of these methods has been examined in a $180 \mathrm{~m}$-long moving train model testing system. The speed of the trailer alone reaches up to $490 \mathrm{~km} \mathrm{~h}^{-1}$. Consequently, a $34.8 \mathrm{~kg}$ model accelerates up to $350 \mathrm{~km} \mathrm{~h}^{-1}$; the smooth and safe stopping of the model is also possible.
\end{abstract}

moving train model, acceleration, deceleration, magnetic braking, air gun, compressed air

Citation: Yang Q S, Song J H, Li D, et al. Train model acceleration and deceleration. Sci China Tech Sci, 2013, 56: 642-647, doi: 10.1007/s11431-012-5101-5

\section{Introduction}

A train model (TM) is accelerated to a defined speed within a limited distance in a moving train model testing system (MTMTS) to investigate the aerodynamic performance of high-speed train configurations [1-3]. After the testing section (TS), the moving TM enters the deceleration section. Afterward, the TM begins to decelerate until it finally stops. For a successful MTMTS, powerfully driving heavy TMs with large dimensions to a speed higher than $300 \mathrm{~km} \mathrm{~h}^{-1}$ is essential. The cross dimension and the aerodynamic configuration of TMs should not be restricted by the MTMTS structure. And, a pantograph is installed on the top of the TM to achieve an aerodynamic simulation of a train with a high speed. Within our knowledge [1-5], however, there has not any published paper on the experimental study for a TM with pantograph model.

\footnotetext{
*Corresponding author (email: qsyang@imech.ac.cn)
}

Two methods are available to accelerate TMs. The first method utilizes an elastic rope to produce kinetic energy for the TM [1]. The elastic rope is elongated, thereby storing potential energy. The release of the elastic rope provides much energy, which causes TM acceleration. Considering the limited contracting speed of the rope, a set of traveling pulleys is introduced, and the dragline of the TM is twisted on the wheels of the pulley. Thus, the speed of the TM is several times greater than that of the contraction of the elastic rope. This multiple depends on the structure of the pulley. Too much friction occurs between the wheels and the dragline. Moreover, the rotation of these wheels, which have great angular speeds, consumes a large proportion of kinetic energy from the elastic rope. Therefore, this technology is only effective for accelerating heavy TMs at speeds lower than $300 \mathrm{~km} \mathrm{~h}^{-1}$.

Another method of accelerating a TM is the utilization of compressed air. The TM is directly driven by the compressed air in a tube [2-5]. Although the speed of the TM exceeds $300 \mathrm{~km} \mathrm{~h}^{-1}$, this method is unsuited for launching 
heavy TMs with larger dimensions because of the following reasons. First, the cross dimension and the configuration of TMs are limited by the inner size of the launching tubes because TMs glide when accelerating. The dimension of a $\mathrm{TM}$ is proportional to both the inner size of the launching tube and the cost of the experimental device. Second, the installation of the braking device of the TM is also restricted by the launching tube because the TM will decelerate and stop after passing TS. Third, stopping a matching piston, which is used to push the model and seal the compressed air, is difficult at the end of the launching tube because the aerodynamic configuration of the train is experimentally simulated. Fourth, the compressed air flow from the launching tube will seriously disturb the testing process. Although the airflow can be partially eliminated by using a cavity device [5], it is difficult to use this technique in a MTMTS with two TMs simultaneously moving in opposite directions along two parallel tracks that have limited vertical distance. Finally, installing a pantograph model at the top of the TM is complicated because of the launching tube. Therefore, testing the TM with a pantograph is not conducted if the $\mathrm{TM}$ is directly launched from a launching tube.

Stopping a TM after it passes through TS is essential but difficult to conduct because of the high speed of the TM. One way of dissipating the kinetic energy of the TM is to increase the friction between some elements of the TM and the surface [1]. However, a heavy TM moving at a high speed has a large kinetic energy. Thus, the friction required to stop the TM may damage the surfaces of the braking elements and may seriously affect the service life of the TM. Moreover, the braking device has a relatively complex structure. Another way to reduce the kinetic energy of the $\mathrm{TM}$ is to produce resistance from the relative movement of iron elements in the magnetic field. Magnetic fields, which involve non-friction brakes, have been introduced in a variety of deceleration applications [6-10].

A device with a length of $180 \mathrm{~m}$ is established to develop a MTMTS in which a heavy TM with a large dimension will be accelerated to a speed higher than $350 \mathrm{~km} \mathrm{~h}^{-1}$. The accelerating power of this device comes from the compressed air, and the resistance of the TM is provided by the interaction of the magnetic field with the steel plate. Corresponding operating parameters, as well as simple discussions on the device, are given in this paper.

\section{Device structure, theory, and device parameters}

The sketch of the device on the acceleration of the TM caused by the compressed air is shown in Figure 1. The acceleration structure of the TM consists of the trailer, the upper track, the lower track, the towrope, the piston, the acceleration tube, the leak hole, and the deceleration tube, among others. The compressed air is prestored in a gas tank. The sudden release of the compressed air is carried out by an air gun. Airflow enters the acceleration tube and compels the piston to move forward because of the difference of pressures between the two sides of the piston in the tube. During acceleration, the piston tows the trailer forward by using a towrope, and the trailer pushes or pulls the TM on the upper track. The leakage of airflow from the hole of the towrope is ignored because the diameter of this hole is smaller than that of the acceleration tube. After the piston passes through the leak hole, the acceleration of the piston, the towrope, and the trailer will rapidly stop because of the leakage of the airflow through the leak hole. Subsequently, the trailer begins to brake by using the trailer magnetic braking device, as shown in Figure 1(b). At the beginning of the trailer deceleration process, the TM is automatically disengaged with the trailer because of the inertia of the TM and then continues to move forward along the upper track.

Having passed through the acceleration section, the trailer begins to decelerate due to the dissipation of the kinetic energy. Thus, the piston, the towrope, and the trailer gradually slow down and finally stop. The magnetic braking device for the trailer includes cylindrical permanent magnets, as shown in Figure 2, which are embedded on the magnet container, and the steel plates on both sides of the magnet container. All the magnets have similar pole orientations. Relative movement occurs between the steel plates and the trailer during trailer braking.

Another type of magnetic braking device is utilized to decrease the speed of the TM in a stable manner in Figure 3. The resistance of the magnetic braking device comes from the relative movement of the magnetic field, which is also provided by the cylindrical magnets with steel plates. The $\mathrm{N}$ and $\mathrm{S}$ poles of all the magnets have similar orientation alignments. Based on the arrangements of the magnets in Figures 2 and 3, the brake force of the first device is stronger than that of the second as the magnets of the two devices have the same mass. The magnetic fields of the both sides of the magnets are all utilized in the trailer magnetic braking device, but only one side is useful in the TM magnetic braking device, thereby causing the difference in the braking forces of the two braking devices.

A formula to describe the transformation of the energy between the compressed air and the kinetic energy of the moving elements has been established. Because the power from the compressed air makes the piston, the towrope, the trailer, and the TM accelerate, the corresponding formula is expressed as follows:

$$
\eta \int_{0}^{L} 10^{5} P S g \mathrm{~d} l=\frac{1}{2}(M+m) v^{2},
$$

where $S$ and $L$ are respectively the cross-section area of the piston and the length of the acceleration section, their units are $\mathrm{m}^{2}$ and $\mathrm{m}$, respectively. $P$ is the pressure on the piston and with a unit of MPa, and $M$ and $m$ are the masses of the trailer assembly, which includes the trailer, the towrope, the 

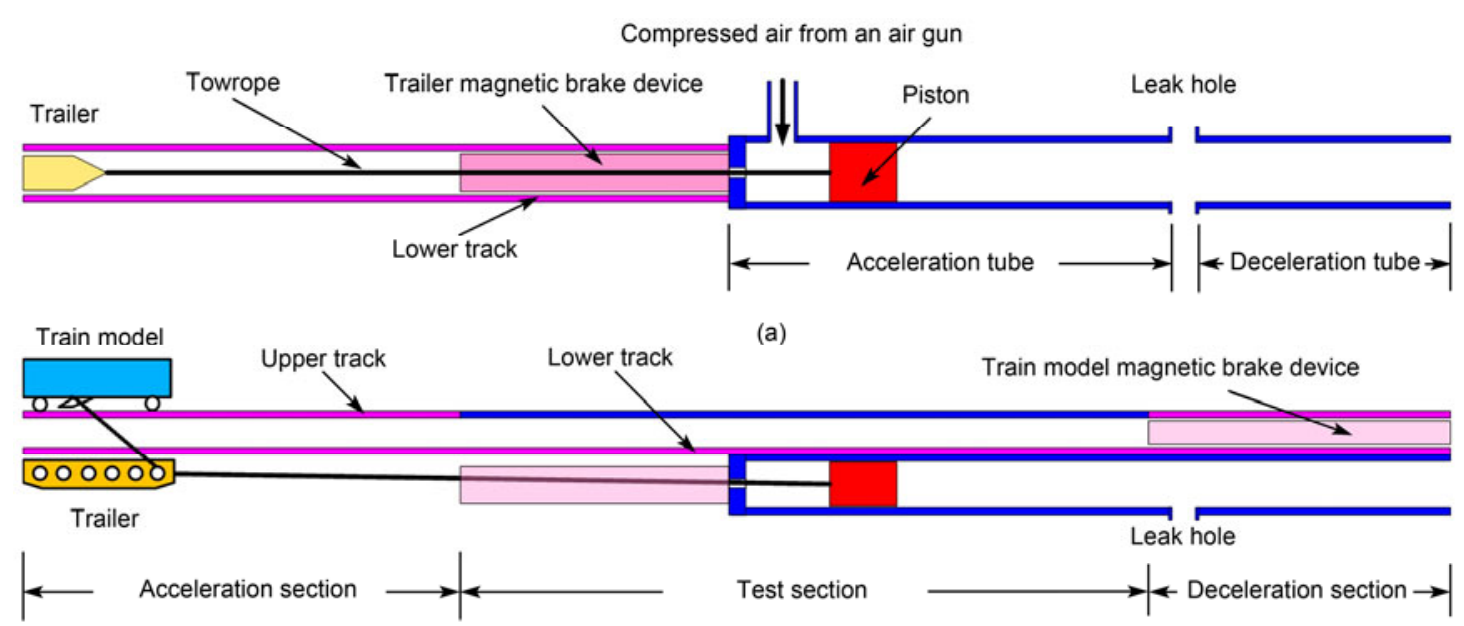

(b)

Figure 1 Sketch of the acceleration of the trailer and TM. (a) Piston and the trailer in the bottom; (b) side view of the MTMTS.

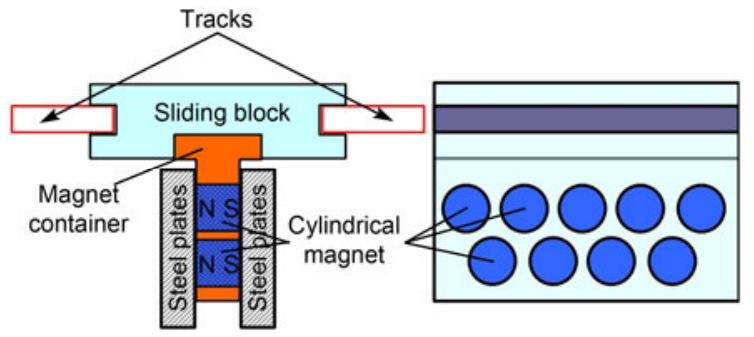

Figure 2 Structure sketch of the trailer magnetic braking device where $\mathrm{N}$ and $\mathrm{S}$ are the two poles of the cylindrical magnets in the magnet container.

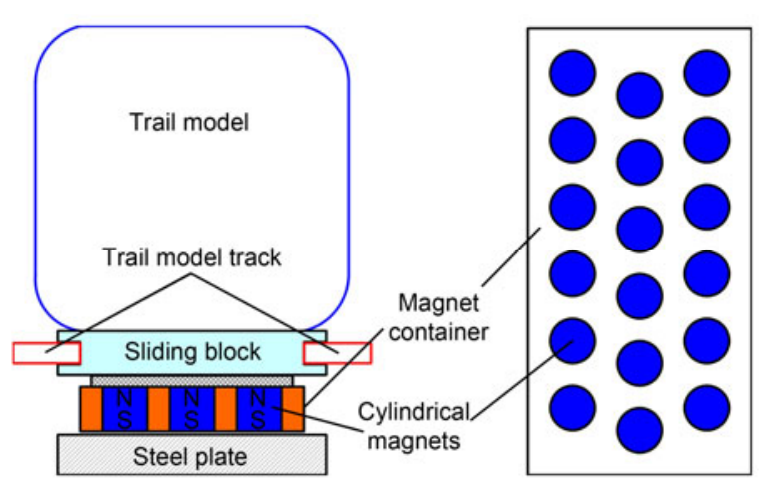

Figure 3 Structure sketch of the TM magnetic braking device where N and $\mathrm{S}$ are the two poles of the cylindrical magnets in the magnet container.

piston, and the TM and their units are all $\mathrm{kg}$. Moreover, $v$ is the speed of the trailer assembly at the end of the acceleration section with a unit of $\mathrm{m} \mathrm{s}^{-1}$, The parameter $\eta$ is the transformation efficiency of the potential energy, which is stored in the compressed air, into the kinetic energy of the moving elements, and $g$ is the gravitational constant with a value of 9.8 .

Assuming that air is an ideal gas and follows the equation $P V=P_{0} V_{0}$, and the expansion of the compressed air fol- lows an isothermal process, a formula for the transformation of energy from the compressed air to the moving elements can then be obtained and expressed as follows:

$$
\eta 10^{5} P_{0} V_{0} g \ln \left(1+\frac{S L}{V_{0}}\right)=\frac{1}{2}(M+m) v^{2},
$$

where $P_{0}$ and $V_{0}$ are the pressure and volume of the compressed air at the beginning of the process, respectively, and the unit of $V_{0}$ is $\mathrm{m}^{3} . P$ and $V$ are the corresponding variables in the acceleration process, respectively.

In our MTMTS, the accelerating section and the accelerating tube both are $40 \mathrm{~m}$ long. The lengths of the trailer magnetic braking device and the decelerating tube are both $45 \mathrm{~m}$, and the inner diameter of each tube is $110 \mathrm{~mm}$. The gross weight of the trailer, the towrope, and the piston is $22.1 \mathrm{~kg}$, and the weight of a TM is $34.8 \mathrm{~kg}$. The volumes of the two storage tanks for the compressed air are 0.3 and $0.8 \mathrm{~m}^{3}$. The gap of the two steel plates in the trailer magnetic braking device is $34 \mathrm{~mm}$. The length, thickness, and height of the trailer are 840, 34, and $96 \mathrm{~mm}$, respectively. The diameter of the towrope is $1 \mathrm{~cm}$. The total weights of all the magnets on the trailer and TM are $7.2 \mathrm{~kg}$ and $12.0 \mathrm{~kg}$, respectively. In this MTMTS, the parameters for eq. (2) are $S=0.0095 \mathrm{~m}^{2}, L=40 \mathrm{~m}, g=9.8 \mathrm{~m} \mathrm{~s}^{-2}, M=22.1 \mathrm{~kg}$, and $V_{0}=0.3$ or $0.8 \mathrm{~m}^{3}$. The efficiency $\eta$ is determined by the structure of the MTMTS, and the speed $v$ depends on the initial pressure $P_{0}$ of the air gun.

\section{Experimental results and discussion}

To obtain the evolution of the acceleration and speed of the trailer and the TM, an acceleration sensor (ULT27100) with a measuring range of $\pm 200 \mathrm{~g}$ and a corresponding data acquisition instrument (DATAQ DI710), which is used to 
record the acceleration of the trailer or TM, is arranged in the trailers or the TM. The evolution of the corresponding speed is induced by using the data on the acceleration.

The trailer alone is accelerated by the compressed air from the air gun. The dependence of the maximum speed on the initial pressure is shown in Figure 4, in which the volume of the compressed air in the air gun is $0.3 \mathrm{~m}^{3}$. The speed increases rapidly with the increasing of the initial pressure as the pressure is low. Moreover, the rate of increasing speed decreases when the pressure increases. This phenomenon occurs because the kinetic energy is linearly proportional to the work from the compressed air, as shown in eq. (2).

We use eq. (2) to analyze the efficiency of the system based on the experimental results shown in Figure 4. The energy conversion efficiency $\eta$ of the air gun with increasing initial pressure $P_{0}$ is shown in Figure 5. The efficiency of the system is low when the pressure is low, and the efficiency of the system gradually becomes constant with increasing $P_{0}$. This occurs because the force used to overcome the friction between the piston and the wall of the acceleration tube and the friction between the sliding blocks on the trailer and the tracks by the compressed air is of a high percentage of the overall force of the compressed air when $P_{0}$ is low. This percentage gradually decreases with increasing

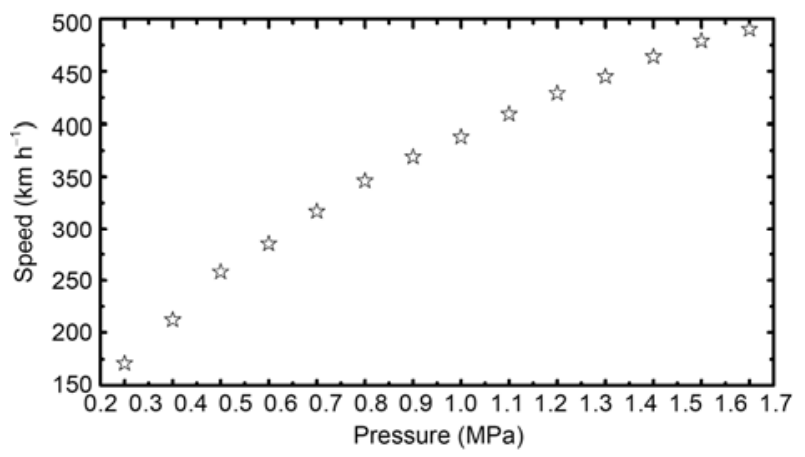

Figure 4 Dependence of the maximum speed of the trailer without the $\mathrm{TM}$ on the initial pressure of the air gun where the volume of the compressed air is $0.3 \mathrm{~m}^{3}$.

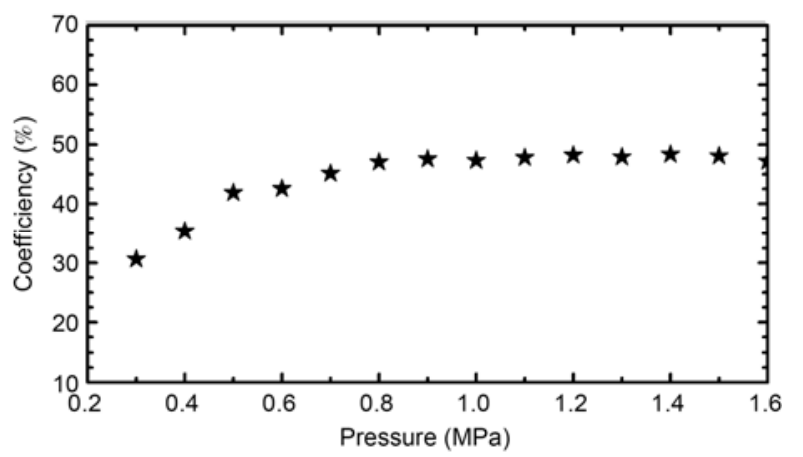

Figure 5 Dependence of the transformation efficiency on the pressure of the air gun where the volume of the compressed air is $0.3 \mathrm{~m}^{3}$.
$P_{0}$. The conversion coefficient tends to become constant with increasing pressure because the thrust from the compressed air is much larger than the friction.

The trailer assembly, which includes the trailer, the towrope, and the piston, has high kinetic energy after the acceleration process is completed. For example, the value of the trailer assembly with a speed of $360 \mathrm{~km} \mathrm{~h}^{-1}$ and a mass of $20 \mathrm{~kg}$ is approximately $100 \mathrm{~kJ}$, and the average damping force is $2500 \mathrm{~N}$ if the braking distance is $40 \mathrm{~m}$. The structure shown in Figure 2 can provide a high level of braking force. The relationship of the deceleration distance with the maximum speed of the trailer is shown in Figure 6, which corresponds to the data shown in Figure 4. In addition, Figure 7 shows the corresponding braking force versus speed. The average braking force is relatively small when the pressure is low, and the damping force gradually rises with increasing speed because the lower the speed of the trailer and the smaller the Lorentz force due to the movement of the magnets in the trailer magnet braking device. The average braking force is $4200 \mathrm{~N}$ when the speed is higher than $300 \mathrm{~km} \mathrm{~h}^{-1}$

After the acceleration section, the trailer reaches its maximum speed and then begins to enter the trailer magnetic braking section. The typical evolution of the acceleration of the trailer is shown in Figure 8(a). The acceleration value of the trailer is slightly higher at the beginning of the acceleration and then gradually decreases because of the

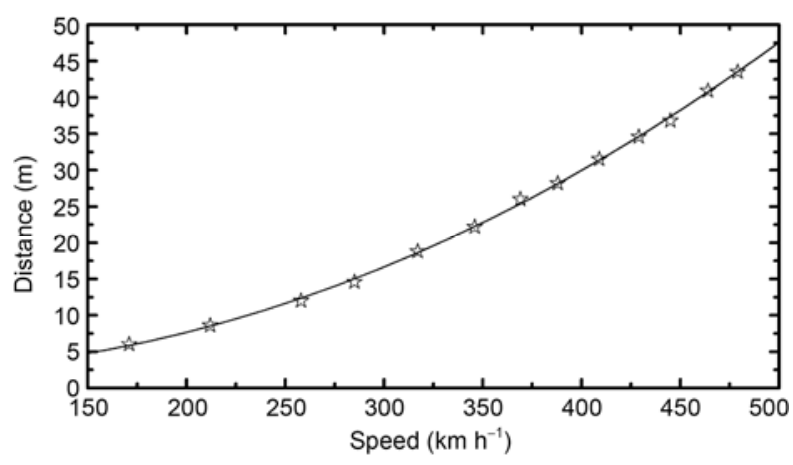

Figure 6 Braking distance versus the maximum speed of the trailer.

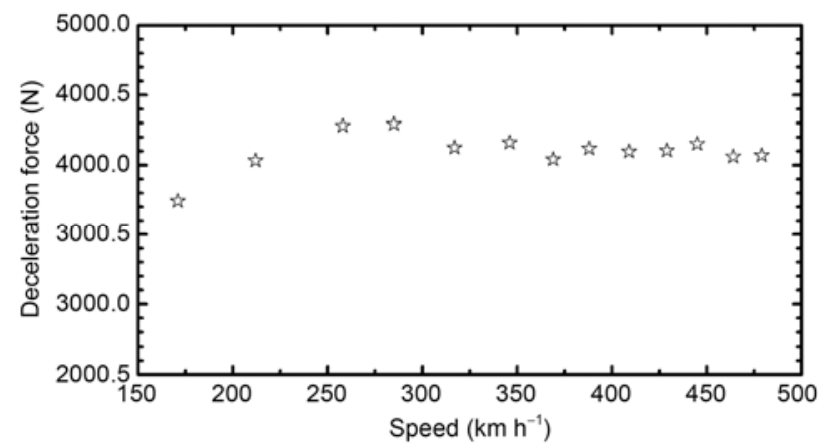

Figure 7 Average values of the braking force with the maximum speed of the trailer. 

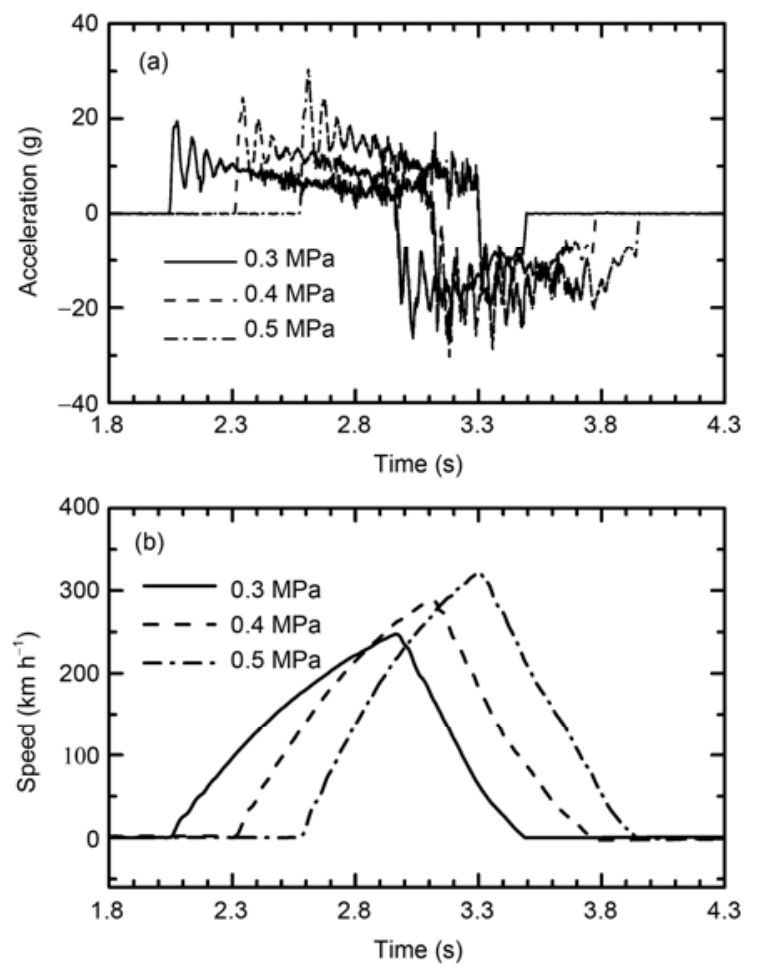

Figure 8 Evolution of the speed of the trailer without the TM, where the volume of the air gun for the compressed air is $0.8 \mathrm{~m}^{3}$.

expansion of the compressed air in the acceleration tube. Another interesting phenomenon is the regular oscillation of acceleration in the whole process, which is caused by the towrope between the piston and the trailer. At the beginning of the acceleration, the sudden acceleration of the piston results only in a high acceleration of the towrope because of the limited elongation of the towrope. Therefore, the tension wave propagates forward and backward along the towrope, which results in acceleration fluctuation at the start of the process. This phenomenon gradually disappears with the expansion of the compressed air. The corresponding speed curves to the evolutions in acceleration in Figure 8(a) are shown in Figure 8(b). The evolutions in speed are smooth although some fluctuations in the acceleration occur at the beginning of the acceleration section. Thus, the influence of the acceleration fluctuation on the speed can be ignored.

When the TM is also accelerated, the measuring instrument is installed on the TM. Thus, the acceleration and speed in the whole test process are measured and deducted. The maximum speed of the TM versus the initial pressure of the air gun is shown in Figure 9. Experimental results reveal that a TM with a mass of $34.8 \mathrm{~kg}$ can be accelerated to a speed of $350 \mathrm{~km} \mathrm{~h}^{-1}$ or even higher when the pressure of the air gun reaches $1.424 \mathrm{MPa}$.

A typical acceleration curve of the TM versus time is shown in Figure 10(a). The figure indicates that some similar cases in fluctuations of acceleration without the TM exist at the beginning of the acceleration process. Fluctuations in acceleration are stronger in the case with the TM than

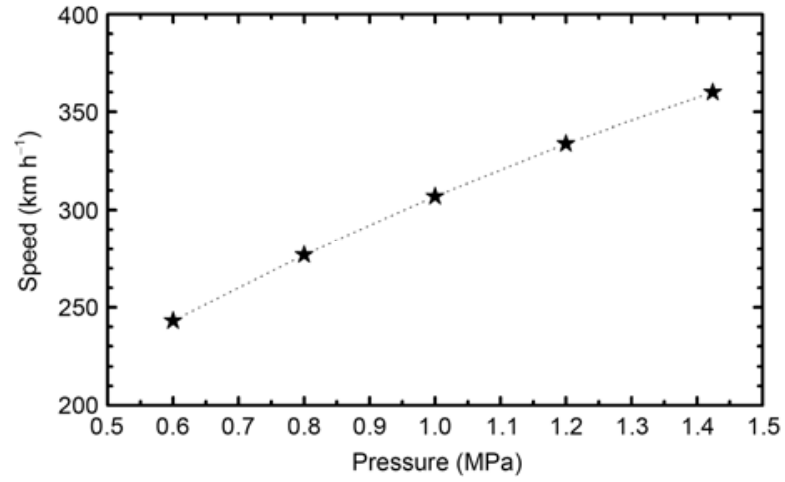

Figure 9 Dependence of the speed of the TM on the initial pressure of the air gun, where the volume of the compressed air is $0.8 \mathrm{~m}^{3}$.
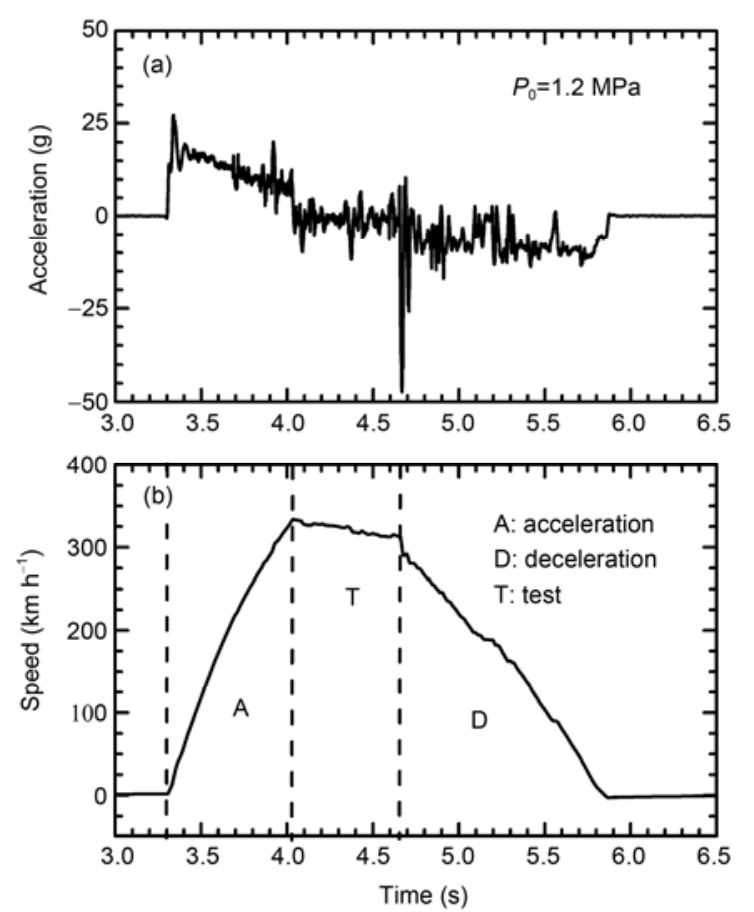

Figure 10 Evolution of (a) the acceleration and (b) the speed of the TM, where the volume of the air gun for the compressed air is $0.8 \mathrm{~m}^{3}$ and the initial pressure of the air gun is $1.2 \mathrm{MPa}$.

that of the trailer alone because of the low smoothness of the upper track. Moreover, the arrangement of the steel plates for the deceleration of the TM for the magnet brake is discontinuous, which corresponds to the apparent acceleration jump at the speed horizontal state, as shown in Figures 10(a) and 10(b). These shortcomings will be eliminated when the smoothness of the tracks is improved and when the steel plates on the floor are set up continuously for the deceleration of the TM.

\section{Conclusion}

Methods on the acceleration and deceleration of a TM with 
a large mass by using compressed air from an air gun and magnetic braking devices are proposed and examined. Based on the principles above, a device with a length of $180 \mathrm{~m}$ was established for the acceleration, test, and deceleration of the TM. A TM with a mass of $34.8 \mathrm{~kg}$ could be accelerated to higher than $350 \mathrm{~km} \mathrm{~h}^{-1}$ and could be safely decelerated and stop.

The work was supported by the National Key Technology R\&D Program (Grant No. 2009BAG12A03) and the National Natural Science Foundation of China (Grant No.10472123).

1 Tian H Q. Train Aerodynamics (in Chinese). Beijing: China Railway Publish House, 2007. 99-114

2 Jiang Z L, Matsuoka K, Sasoh A, et al. Numerical and experimental investigation of wave dynamic processes in high-speed train/tunnels.
Acta Mech Sin, 2002, 18: 209-226

3 Takayama K, Sasoh A, Onodera O, et al. Experimental investigation on tunnel sonic boom. Shock Waves, 1995, 5: 127-138

4 Sasoh A, Matsuoka K, Nakashio K, et al. Attenuation of weak shock waves along pseudo perforated walls. Shock Waves, 1998, 8: 149159

5 Demmenie E, De Bruin A, Klaver E. Experimental pressure wave research at NLR for high-speed rail tunnels, 1998, NLR Technical Publication TP98375

6 Jirout M, Mack W, Lugner P. Non-smooth dynamics of a magnetic track brake. Regul Chaotic Dyn 2009, 14: 673-681

7 Ma J, Zhang B, Huang X,et al. Design and analysis of the hybrid excitation rail eddy brake system of high-speed trains. J Zhejiang Univ-Sci A 2011, 12: 936-944

8 Jou M, Shiau J, Sun C. Design of a magnetic braking system. J Magn Magn Mater, 2006, 304: e234-e236

9 Maclatchy C, Backman P, Bogan L. A quantitative magnetic braking experiment. Am J Phys, 1993, 60: 1096-1101

10 Ma D, Shiau J. The design of eddy-current magnet brakes. T Can Soc Mech Eng, 2010, 3: 19-37 